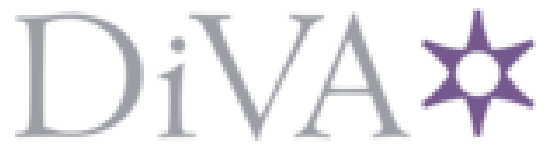

http://www.diva-portal.org

This is the published version of a paper published in Journal of Physical Chemistry Letters.

Citation for the original published paper (version of record):

Lundberg, P., Wei, Q., Ge, Z., Voit, B., Reineke, S. et al. (2020)

Polymer Featuring Thermally Activated Delayed Fluorescence as Emitter in Light-

Emitting Electrochemical Cells

Journal of Physical Chemistry Letters, 11(15): 6227-6234

https://doi.org/10.1021/acs.jpclett.oc01506

Access to the published version may require subscription.

N.B. When citing this work, cite the original published paper.

Permanent link to this version:

http://urn.kb.se/resolve?urn=urn:nbn:se:umu:diva-170537 


\title{
Polymer Featuring Thermally Activated Delayed Fluorescence as Emitter in Light-Emitting Electrochemical Cells
}

\author{
Petter Lundberg, Qiang Wei,* Ziyi Ge, Brigitte Voit, Sebastian Reineke, and Ludvig Edman*
}

Cite This: J. Phys. Chem. Lett. 2020, 11, 6227-6234

Read Online

ABSTRACT: Semiconducting polymers that feature thermally activated delayed fluorescence (TADF) can deliver a much desired combination of high-efficiency and metal-free electroluminescence and cost-efficient solution-based fabrication. A TADF polymer is thus a very good fit for the emitting compound in light-emitting electrochemical cells (LECs) because the commonly employed air-stabile and fewlayer LEC architecture is well suited for such solution-based fabrication. Herein we report on the first LEC device based on a TADF polymer as the emitting species, which delivers a luminance of $96 \mathrm{~cd} \mathrm{~m}^{-2}$ at $4 \mathrm{~V}$ and a current efficacy of $1.4 \mathrm{~cd} \mathrm{~A}^{-1}$ and $>600 \mathrm{~cd} \mathrm{~m}^{-2}$ at $6 \mathrm{~V}$, which is competitive with the performance of multilayer organic light-emitting diodes based on the same TADF polymer. We further utilize the established sensitivity of the emission of the TADF polymer to its environment to draw conclusions on the exciton populations in host-guest and host-free TADF LEC devices.

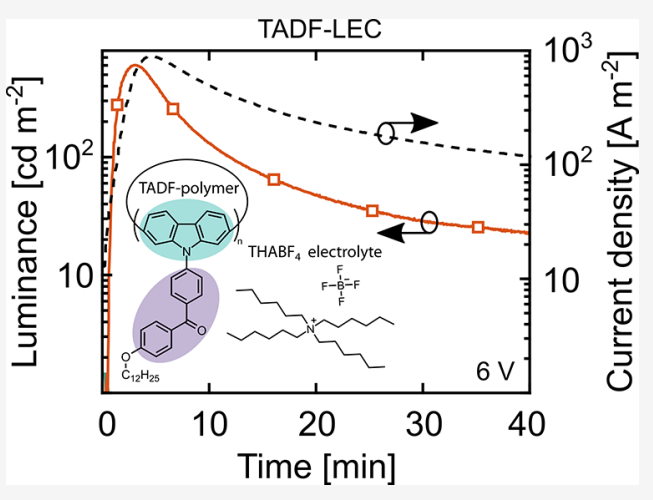

$\mathrm{T}^{\mathrm{k}}$ he emergence of thin, flexible, and area-emissive technologies is projected to open up for new and functionalized applications of light in, for example, medicine $e^{1,2}$ and security. ${ }^{3}$ The frontrunner in the area-emissive field is the organic light-emitting diode (OLED). At industrial grade, OLEDs widely adopt a multitude of different layers, which are deposited with nanometer precision under high vacuum. As such, the OLED is, and will probably remain, expensive, which prohibits its use in several of the emerging applications. In this context, the light-emitting electrochemical cell (LEC) is an interesting alternative. ${ }^{4-9}$ The LEC can also feature conformable areal emission, but, by the virtue of its in situ doping operational mechanism, ${ }^{10-14}$ it can be fabricated with low-cost solution-based printing and coating methods under ambient air. ${ }^{5,15-17}$

However, a current drawback is that today's most efficient LECs (and OLEDs) comprise phosphorescent organic semiconducting emitters, which are based on expensive and rare heavy metals from the platinum group in the periodic table. ${ }^{18-24}$ This is because excitons (bound electron-hole pairs) form in a $\sim 3: 1$ triplet/singlet ratio in organic semiconductors during electric driving, ${ }^{25,26}$ and it is only such heavy-metal-based phosphorescent emitters that exhibit efficient and high-brightness electroluminescence (EL) from the triplet state with a reasonable excited-state lifetime in the range of a few microseconds due to strong spin-orbit coupling. In addition, the singlet excitons in phosphorescent emitters can be efficiently transferred to the triplet state by intersystem crossing (ISC), so that, in principle, all electrically generated excitons can be harvested for light emission. ${ }^{27}$
A recently invented class of heavy-metal free organic compounds addresses the above issue through the emission process of thermally activated delayed fluorescence (TADF).$^{28-32}$ Efficient TADF emitters are designed so that the highest occupied molecular orbital (HOMO) and the lowest unoccupied molecular orbital (LUMO) are spatially separated, which render the lowest excited singlet state $\left(S_{1}\right)$ and the lowest excited triplet state $\left(\mathrm{T}_{1}\right)$ positioned close in energy; with such a design, a thermally promoted reverse intersystem crossing (RISC) from the nonemissive $T_{1}$ to the emissive $S_{1}$ can take place. ${ }^{33}$ Accordingly, all of the electrically generated singlet and triplet excitons can in a TADF emitter be utilized for light emission via prompt and delayed fluorescence, respectively.

A vast majority of today's TADF emitters are small molecules, which commonly are best fit for deposition by (expensive) physical vapor deposition under high vacuum. In contrast, polymers and oligomers allow for facile solutionbased processing, but the number of such materials exhibiting TADF emission is very limited, in part due to a complicated synthesis protocol, ${ }^{34-41}$ and, so far, no LEC device based on an oligomeric or polymeric TADF emitter has been reported.

Received: May 15, 2020

Accepted: July 6, 2020

Published: July 6, 2020 

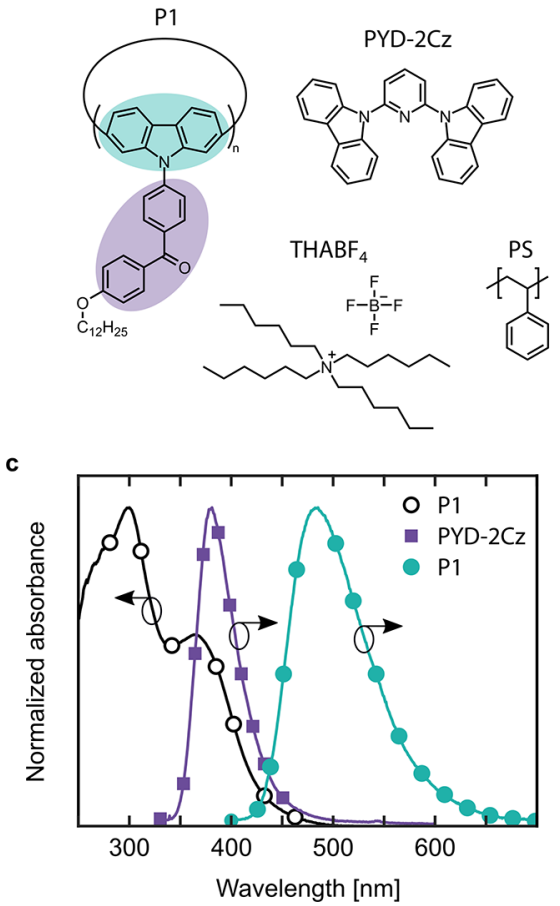

b

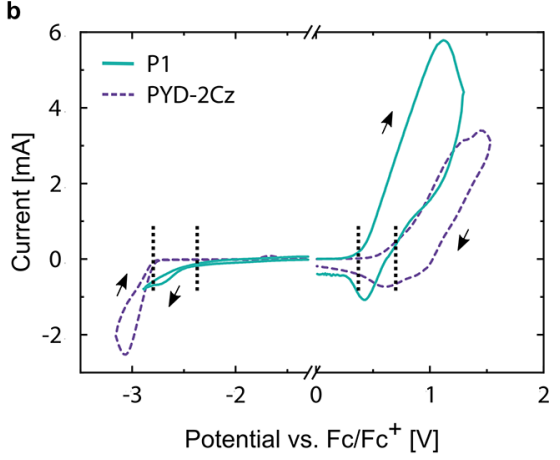

d

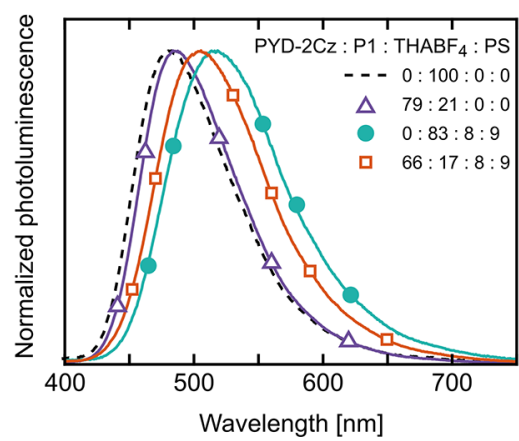

Figure 1. (a) Chemical structure of the active-material constituents: the P1 polymeric TADF emitter, the PYD-2Cz host compound, the THABF 4 electrolyte, and the PS additive. The electron-donor unit of P1 is marked with a cyan background color, and the electron-acceptor unit is marked with a purple background color. (b) Cyclic voltammetry traces recorded on thin films of P1 (solid cyan line) and PYD-2Cz (dashed purple line). The vertical dotted lines indicate the onset potentials for oxidation and reduction. The scan speed was $0.05 \mathrm{~V} \mathrm{~s}^{-1}$. (c) Normalized absorbance of a thin film comprising P1 dispersed in PS (open black circles; note that the contribution of PS is effectively eliminated by the measurement procedure) and the normalized photoluminescence (PL) of neat films of $\mathrm{PYD}-2 \mathrm{Cz}$ (solid purple squares) and P1 (solid cyan circles). (d) Normalized PL of different active-material films, with the mass ratio of the constituents defined in the inset.

Herein we address this issue and report on the pioneering realization of functional three-layer LEC devices based on a polymeric TADF emitter, which turn on and emit light at a low voltage below $4 \mathrm{~V}$ and which emit with a bright luminance of $>600 \mathrm{~cd} \mathrm{~m}^{-2}$ at $6 \mathrm{~V}$. The operation of LEC devices is intrinsically dependent on the existence of mobile ions in the active material, and we further establish that the emissive state of the polymeric TADF emitter, because of its intramolecular charge-transfer character, is highly sensitive to the proximity of the polar ions. With this information in hand, we are able to draw conclusions regarding the exciton profiles that are present in different LEC devices during operation, and we notably find that the excitons can be self-trapped at ion-induced low-energy sites positioned at the edges of the emission zone.

Figure 1a presents the chemical structure of the TADFactive macrocycle conjugated polymer P1, which was synthesized by a Yamamoto-type polymerization of the nonTADF monomer (4-(3,6-dibromo-carbazol-9-yl)phenyl)(4(dodecyloxy)phenyl)methanone. ${ }^{42} \mathrm{P} 1$ is designed to feature a twist between the electron-donating carbazole unit (marked with a cyan background) and the electron-accepting benzophenone unit (marked with a purple background), which results in the HOMO positioned on the carbazole being spatially well separated from the LUMO localized on the benzophenone. The appearance of TADF emission from the P1 polymer, but not the constituent monomer, originates in a conjugation-induced reduction of the energy difference between the $S_{1}$ and $T_{1}$ states while keeping the radiative $S_{1}-$ $S_{0}$ decay rate fast. ${ }^{42}$ The dodecyloxy end group was included for enhanced solubility in common organic solvents, such as toluene, tetrahydrofuran, and chloroform.

It is a common practice to disperse TADF (and triplet) emitters as a minority guest in a larger energy-gap host matrix because this inhibits the diffusion of the long-lived triplet excitons and thereby suppresses nondesired triplet-triplet and triplet-polaron quenching interactions. The host-guest design is also beneficial because it lowers the self-absorption of the guest-generated light within the active material. The drawback is that the guest emitter can function as a trap for electron and hole transport, especially in thick host-guest layers. Herein we have, after careful screening (following the criteria detailed later), selected to employ 2,5-di(9H-carbazol-9-yl)pyridine (PYD-2Cz) as the host compound for the P1 guest emitter. The active material in LEC devices must, by definition, comprise an electrolyte, and the ionic liquid tetrahexylammonium tetrafluoroborate $\left(\mathrm{THABF}_{4}\right)$ was employed because of its broad electrochemical stability window ${ }^{43}$ and because it has demonstrated good LEC performance in previous studies. A small amount of poly(styrene) (PS) was finally included in the LEC active material because it was found to render the device performance more repeatable in that it effectively eliminated problems with electrical short circuits. The chemical structures of the active-material constituents are displayed in Figure 1a.

The active material (or rather the organic semiconductor) in a functional LEC is electrochemically p-type-doped (oxidized) at the anode and n-type-doped (reduced) at the cathode during operation, so that a light-emitting $\mathrm{p}-\mathrm{n}$ junction doping structure can form at the steady state. We investigated whether this basic doping requirement was fulfilled with cyclic 
voltammetry $(\mathrm{CV})$. Figure $1 \mathrm{~b}$ shows $\mathrm{CV}$ traces of thin films of the TADF emitter P1 (solid cyan line) and the host PYD-2Cz (dashed purple line). We observe that both P1 and PYD-2Cz feature significant and partially reversible oxidation reactions with onsets (as indicated by the vertical dotted lines in Figure 1b) at +0.4 and $+0.7 \mathrm{~V}$ vs $\mathrm{Fc} / \mathrm{Fc}^{+}$, respectively. The reduction reactions were of smaller magnitude and less reversible, and their onsets were located at -2.4 and $-2.8 \mathrm{~V}$ vs $\mathrm{Fc} / \mathrm{Fc}^{+}$for P1 and $\mathrm{PYD}-2 \mathrm{Cz}$, respectively. We mention that at least two prescans were required before the reduction of $\mathrm{P} 1$ could be observed. These results imply that the facile electrochemical ptype doping of both the P1 emitter and the PYD- $2 \mathrm{Cz}$ host can be expected during LEC operation, but they raise some concerns regarding the efficiency and the extent of the n-type doping reaction. ${ }^{44}$

The HOMO and LUMO energy levels can be calculated from the $\mathrm{CV}$-derived onset potentials for oxidation and reduction, respectively, using the equation: $E_{\text {HOMO/LUMO }}$ $(\mathrm{eV})=-\left(4.8 \mathrm{eV}+e V_{\mathrm{Fc}^{\mathrm{ox}} / \mathrm{Fc}^{+}}^{\mathrm{ox} /{ }^{+}}\right)^{45}$ Table $\mathrm{S} 1$ shows that the derived HOMO and LUMO levels for P1 are -5.2 and -2.4 $\mathrm{eV}$, respectively, whereas the corresponding values for PYD$2 \mathrm{Cz}$ are -5.5 and $-2.0 \mathrm{eV}$. This results in the electrochemical energy gap being $2.8 \mathrm{eV}$ for P1 and $3.5 \mathrm{eV}$ for PYD-2Cz. The optical energy gap, as derived from the onset of absorption, is $2.6 \mathrm{eV}$ for P1 (see Figure 1c) and $3.5 \mathrm{eV}$ for PYD-2Cz. ${ }^{46}$

With the energy-level data in hand, the conclusion is that an intermolecular charge-transfer (or exciplex) state between the host and guest will not form in the PYD-2Cz:P1 host-guest system. Specifically, we find that the LUMO offset (or electron trap depth) for the PYD-2Cz:P1 host-guest active material is $0.4 \mathrm{eV}$, whereas the HOMO offset (or hole trap depth) is 0.3 $\mathrm{eV}$. It is noted that such balanced values for the electron and hole trap depths have been reported to facilitate the efficient operation of host-guest LECs. ${ }^{43}$

Figure 1c presents the absorption spectrum of a thin film comprising P1 diluted in PS at 10 mass \% (left $y$-axis) and the photoluminescence (PL) spectra of neat PYD-2Cz and P1 thin films (right $y$-axis). We observe that the PL emission of the PYD-2Cz host (solid purple squares) significantly overlaps the absorption of the P1 guest (open black circles), which demonstrates that a principle requirement for Förster resonance energy transfer (FRET) from PYD-2Cz to $\mathrm{P} 1$ is fulfilled. ${ }^{47}$ The substantial separation, and the lack of overlap, between the PL spectrum of the emitter P1 (solid cyan circles) and the absorption spectrum of the host PYD-2Cz with an onset at $350 \mathrm{~nm}^{46}$ signals that the self-absorption within a thin layer of active material should be low.

The triplet energy of PYD-2Cz is reported to be $2.93 \mathrm{eV}{ }^{46}$ Because the singlet energy of $\mathrm{P} 1$ is $2.6 \mathrm{eV}$ and because the singlet and triplet energies are essentially identical in TADF emitting materials by design, the conclusion is that Dexter energy transfer from PYD-2Cz to P1 should also be an effective population pathway. ${ }^{48}$

Figure 1d and Figure S1 present PL spectra of a number of different thin films comprising the P1 polymer. The neat P1 film exhibits a featureless broad PL spectrum, with its emission peak at $485 \mathrm{~nm}$ (Figure 1d, dashed black line). The corresponding values for the PL quantum yield (PLQY) of the neat $\mathrm{P} 1$ film are $33 \%$ in inert $\mathrm{N}_{2}$ and $32 \%$ under ambient air. We note that these results are essentially identical to those reported in a previous study on the P1 polymer. ${ }^{49}$

The addition of an insulating polymer to the $\mathrm{P} 1$ polymer in the form of 90 mass \% PS resulted in marginal changes to the
PL emission. Specifically, the PL peak remained at $485 \mathrm{~nm}$, although a minor shoulder appeared at lower energies (Figure S1, solid black line), and the PLQY featured a very minor decrease by 3 to $4 \%$. We rationalize that the retained emission performance of P1 following the addition of PS is due to the fact that the two polymers were poorly mixed and wellseparated within the blend because of the characteristic low entropic gain that results from the mixing of polymers.

In contrast, the inclusion of a majority amount of a semiconducting small-molecule host provoked a much stronger effect on the PLQY. Although the PL spectrum is essentially retained following the addition of 79 mass\% PYD-2Cz (Figure $1 \mathrm{~d}$, open purple triangles), the PLQY almost doubled to $62 \%$ under $\mathrm{N}_{2}$ and increased to $51 \%$ under ambient air. The significant difference between the PLQY under inert $\mathrm{N}_{2}$ and ambient air is in line with the fact that the P1 polymer is a TADF emitter. Specifically, a significant fraction of the initially UV-excited singlets on the $\mathrm{P} 1$ polymer are transferred to the long-lived triplet state by ISC, where they are quenched by $\mathrm{O}_{2}$ molecules in ambient air. Under inert $\mathrm{N}_{2}$, in contrast, the triplets survive for a sufficiently long time so that they can be recycled back to the emissive singlet state by RISC.

We further find that the PYD $-2 \mathrm{Cz}$ host PL, with its peak located at $380 \mathrm{~nm}$ (Figure 1c, solid purple squares), is completely absent in the host-guest blend, which demonstrates that the host-to-guest transfer, as desired, is complete. We also note that significant changes to the P1 emission capacity have been reported following the inclusion of other small-molecule host compounds, although the exact changes appear to be intimately linked to the detailed chemical and energy structure of the host compound. ${ }^{42,49}$ Our conclusion is that the PYD$2 \mathrm{Cz}$ host and the P1 guest interact strongly in the host-guest blend, as manifested in the fact that the host-to-guest transfer is complete and the PLQY of P1 increases strongly, and we suggest that the majority PYD-2Cz host acts as a solid-state solvent for the minority $\mathrm{P} 1$ guest. $^{50}$

The most dramatic spectral effect is, however, observed following the addition of a small amount (8 mass \%) of $\mathrm{THABF}_{4}$ electrolyte (and 9 mass \% inert PS); see Figure 1d, solid cyan circles. The PL peak is red-shifted by $33 \mathrm{~nm}$ with respect to the neat P1 film, whereas the PLQY is $47 \%$ under $\mathrm{N}_{2}$ and $41 \%$ under ambient air. Figure S2 shows that the inclusion of the $\mathrm{THABF}_{4}$ electrolyte has a negligible effect on the absorption spectrum. Combined, these results therefore imply that the polar $\mathrm{THABF}_{4}$ electrolyte can interact strongly with the P1 emitter, but the effect of this interaction is primarily manifested in the excited state of P1 (as detected with PL) and not in its ground state (as probed with absorption). We note that previous studies on TADF emitters report on a similar increasing red shift of the PL spectrum with increasing polar (solution) environment, which is in line with the fact that the excited state of TADF emitters exhibits an intramolecular charge-transfer character. ${ }^{50-52}$

The combined addition of both the PYD-2Cz host and the $\mathrm{THABF}_{4}$ electrolyte (and the PS polymer) to the P1 film resulted in the highest PLQY values and a PL spectrum located in between that of the neat host-guest and the neat guestelectrolyte PL spectra. Specifically for the active-material film optimized for LEC operation (Figure 1d, open red squares), featuring a $\left\{\mathrm{PYD}-2 \mathrm{Cz}: \mathrm{P} 1: \mathrm{THABF}_{4}: \mathrm{PS}\right\}$ mass ratio of $\{66: 17: 8: 9\}$, the PLQY is $71 \%$ under $\mathrm{N}_{2}$ and $63 \%$ under ambient air, and the PL peak is located at $505 \mathrm{~nm}$. The general trend with increasing host concentration (at the expense of a 
a

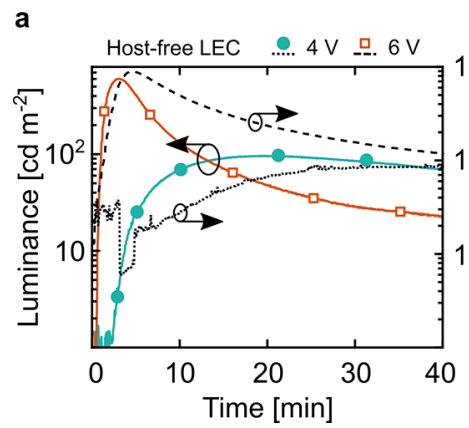

b

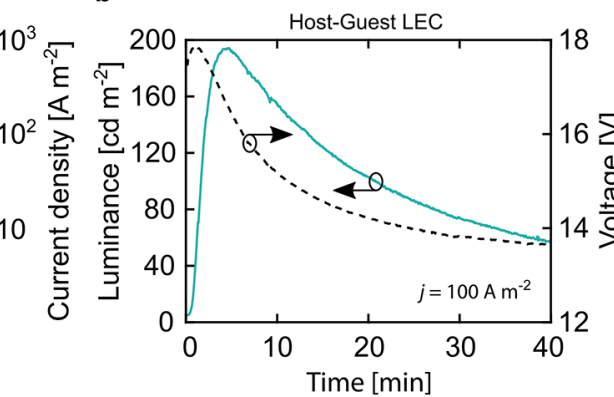

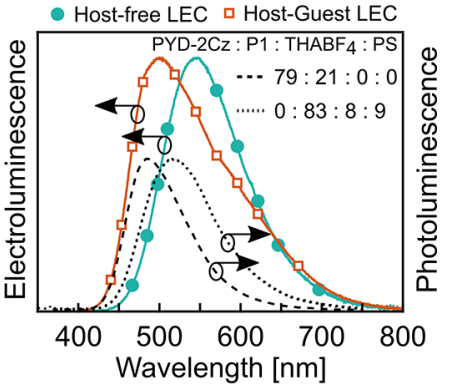

Figure 2. (a) Temporal evolution of the luminance (left $y$-axis) and the current density (right $y$-axis) of ITO/active material/Al host-free LECs during constant-voltage driving at 4 and $6 \mathrm{~V}$. The host-free LECs featured an active-material mass ratio of $\left\{\mathrm{P} 1: \mathrm{THABF}_{4}: \mathrm{PS}\right\}=\{83: 8: 9\}$, and the active-material thickness was $70 \mathrm{~nm}$. (b) Temporal evolution of the luminance (left $y$-axis, solid cyan line) and the voltage (right $y$-axis, dashed black line) for ITO/PEDOT:PSS/active material/Al host-guest LEC during constant current-density driving at $100 \mathrm{~A} \mathrm{~m}^{-2}$. The host-guest LECs featured an active-material mass ratio of $\left\{\mathrm{PYD}-2 \mathrm{Cz}: \mathrm{P} 1: \mathrm{THABF}_{4}: \mathrm{PS}\right\}=\{66: 17: 8: 9\}$, and the active-material thickness was $120 \mathrm{~nm}$. (c) Normalized EL spectrum measured at peak luminance for the host-free LEC (solid cyan circles) and the host-guest LEC (open orange squares). For reference, the PL spectra of the electrolyte-free host-guest film (dashed black line) and the host-free active-material film (dotted black line) are included. The PL excitation wavelength was $300 \mathrm{~nm}$.

lowered guest concentration) is an increase in the PLQY and a blue shift of the PL envelope. (See Figure S1.)

We have systematically evaluated the performance of two distinct types of P1-emitter-based LEC devices: "a host-free LEC" and "a host-guest LEC". During the course of the project, we have tested $>18$ devices in each category, and the device yield was $\sim 50 \%$. We found that the best performance for the host-free LECs was obtained at constant-voltage driving, whereas the host-guest LECs excelled at constantcurrent driving and with a thin layer of poly $(3,4-$ ethylenedioxythiophene):poly(styrene-sulfonate) (PEDOT:PSS) included between the indium-tin-oxide (ITO) anode and the active material. The presented results are for the champion devices, but a similar performance was recorded on at least three additional devices in each category.

Figure 2 a displays the temporal evolution of the luminance (left $y$-axis) and the current density (right $y$-axis) of pristine host-free LECs when driven by a constant voltage of 4 and $6 \mathrm{~V}$. These devices featured a simple three-layer ITO/active material/Al structure, where the optimized $70 \mathrm{~nm}$ thick hostfree $\left\{\mathrm{P} 1: \mathrm{THABF}_{4}: \mathrm{PS}\right\}$ active material exhibited a mass ratio of $\{83: 8: 9\}$. A simultaneous increase in current and luminance was observed during the initial operation, which originates in the mobile-ion-facilitated characteristic in situ electrochemical doping operation of LEC devices that improves the charge injection and renders the active material more conductive. As expected, identical devices void of mobile ions in the active material feature a constant (and much lower) current and no measurable luminance.

The peak luminance at $4 \mathrm{~V}$ is $96 \mathrm{~cd} \mathrm{~m}^{-2}$ at a current density of $66 \mathrm{~A} \mathrm{~m}^{-2}$, corresponding to a current efficacy exceeding 1.4 cd $\mathrm{A}^{-1}$. With the assumption of Lambertian emission, this performance translates to a power efficacy of $1.0 \mathrm{~lm} \mathrm{~W} \mathrm{~W}^{-1}$ at the peak luminance. By increasing the drive voltage to $6 \mathrm{~V}$, the peak luminance increased to $>600 \mathrm{~cd} \mathrm{~m}^{-2}$ but at a lowered current efficacy of $0.9 \mathrm{~cd} \mathrm{~A}^{-1}$. We note that the luminance peaks before the current density, which implies that excitonpolaron quenching interactions start to play a significant role when the fronts of the two doping regions approach each other and the effective width of the $\mathrm{p}-\mathrm{n}$ junction decreases. ${ }^{53}$ The corresponding data for the host-free LEC during driving with a constant current density of $100 \mathrm{~A} \mathrm{~m}^{-2}$ are presented in Figure S3.
We mention that the fluctuating current without light emission during the first few minutes of operation at $4 \mathrm{~V}$ was a relatively common observation for the host-free LECs, which we attribute to the existence and burnoff of minor microshorts through the $70 \mathrm{~nm}$ thin active-material film. An effective means to improve the quality and thickness of the active material was to include the high-solubility PYD $-2 \mathrm{Cz}$ majority host compound in the active material in the host-guest LECs. We found that the best performance for the host-guest LEC was attained with an active-material mass ratio of \{PYD2Cz:P1:THABF $4: P S\}=\{66: 17: 8: 9\}$ and an active-material thickness of $120 \mathrm{~nm}$.

Figure $2 \mathrm{~b}$ displays the temporal evolution of the luminance (left $y$-axis, solid cyan line) and the voltage (right $y$-axis, dashed black line) of such an optimized pristine host-guest ITO/ PEDOT:PSS/active material/Al LEC during driving by a constant current density of $100 \mathrm{~A} \mathrm{~m}^{-2}$. The luminance increased and reached $194 \mathrm{~cd} \mathrm{~m}^{-2}$ after $5 \mathrm{~min}$ of operation, which corresponds to a peak current efficacy of $1.9 \mathrm{~cd} \mathrm{~A}^{-1}$ and a power efficacy of $0.36 \mathrm{~lm} \mathrm{~W} \mathrm{~W}^{-1}$. The voltage, following a short initial increase, consistently decreased during almost $1 \mathrm{~h}$ of operation, which is a direct consequence of the electrochemical doping rendering the active material more conductive. The consistently higher drive voltage for the host-guest LECs compared with the host-free LECs at the same current density is due to the fact that the undoped $p-n$ junction in the former comprises transport-limiting nonfilled trap sites with a trap depth of 0.3 to $0.4 \mathrm{eV}$ (see Table S1) in the form of P1 guest molecules dispersed in the PYD-2Cz host matrix.

We also note that the same TADF-active P1 emitter has been implemented and evaluated in OLEDs, comprising a multitude $(n=3-5)$ of vacuum-evaporated and solutionprocessed functional layers in between the two electrodes. ${ }^{49}$ The structurally much simpler LEC devices in this study were found to be competitive in several performance metrics, and we call particular attention to the lower turn-on voltage and the higher power efficacy at significant luminance $(>100 \mathrm{~cd}$ $\mathrm{m}^{-2}$ ) for the host-free LECs.

Figure $2 c$ presents the normalized EL spectra of the hostfree LEC (solid cyan circles) and the host-guest LEC (open orange squares) recorded at peak luminance. For the hostguest LEC, no emission from the PYD-2Cz host (PL peak = $380 \mathrm{~nm}$, solid purple squares in Figure 1c) can be detected, 
which demonstrates that the host-to-guest energy transfer is complete.

We further find that the spectral differences between the two LEC systems are very large, with the host-free LEC emitting with a relatively narrow EL spectrum with its peak positioned at $547 \mathrm{~nm}$ (Commission Internationale de l'Eclairage (CIE) 1931 color space $x, y$ coordinates $=\{0.39,0.54\})$, whereas the host-guest LEC features a broader and distinctly blue-shifted EL spectrum with its peak emission at $500 \mathrm{~nm}$ (CIE $x, y$ coordinates $=\{0.31,0.44\})$. A closer inspection reveals that the EL spectrum of the host-free LEC appears to originate in one emitting species centered at $547 \mathrm{~nm}$, whereas the EL spectrum of the host-guest LEC appears to be a superposition of two emitting components: a majority species with a peak wavelength of $500 \mathrm{~nm}$ and a minority species with a center wavelength of $\sim 550 \mathrm{~nm}$.

To facilitate the discussion of the origin of these distinct spectral differences, Figure $2 \mathrm{c}$ also presents the PL spectra of the host-guest film void of the $\mathrm{THABF}_{4}$ electrolyte (dashed black line) and the host-free active-material film comprising the same electrolyte (dotted black line). These data remind us that the existence of polar ions in the neighborhood of the P1 emitter is concomitant with a distinct red shift of the emission spectrum, whereas the replacement of the polar electrolyte by the host molecules results in a blue shift of the P1 emission. We also remember that the steady-state operation of an LEC device dictates that all of the mobile ions are locked up in the doping regions as electrostatically compensating counterions. ${ }^{54}$ More specifically, at the steady state, the positive tetrahexylammonium (THA) cations are locked up in the n-type region in between the negative cathode and the $p-n$ junction, whereas the negative $\mathrm{BF}_{4}$ anions are positioned in the p-type region between the positive anode and the emissive $\mathrm{p}-\mathrm{n}$ junction.

Accordingly, it seems plausible that the red-shifted EL from the host-free LEC at steady state is solely taking place from P1 polymers in close proximity to either the THA cations or the $\mathrm{BF}_{4}$ anions located within the doping regions, whereas the twocomponent EL from the host-guest LEC originates from a combination of P1 polymers solely surrounded by host molecules (the majority component at $\sim 500 \mathrm{~nm}$ ) and from P1 polymers in proximity to the polar ions (the minority component at $\sim 550 \mathrm{~nm}$ ).

Our qualitative explanation for these findings is as follows: In the host-free LEC, the excitons are free to diffuse around within the $\mathrm{p}-\mathrm{n}$ junction until they are "trapped" on the lower energy P1 sites that exist close to the ions in the neighboring doping regions, from which they will emit (thus the red-shifted single-emission species). In the host-guest LEC, the exciton diffusion is greatly suppressed because the host compound is the majority component. In this scenario, the excitons will be quickly trapped on the P1 guest sites distributed over the entire $\mathrm{p}-\mathrm{n}$ junction so that the emission will take place from both $\mathrm{P} 1$ sites in the central part of the $\mathrm{p}-\mathrm{n}$ junction that are unaffected by the ions in the doping regions (the majority of the excitons) and from P1 sites close to the doping boundaries that will be affected by the presence of the ions (the minority of the excitons). Accordingly, the observed ion-induced shift of the TADF emission, as exemplified in Figure 1d, can also shed light on the exciton distributions in host-free and host-guest TADF-emitting LEC devices.

To summarize, we report that a novel TADF-emitting polymer can be utilized as the metal-free efficient emitter in
LEC devices, which deliver a luminance of $96 \mathrm{~cd} \mathrm{~m}^{-2}$ at $4 \mathrm{~V}$ and a current efficacy of $1.4 \mathrm{~cd} \mathrm{~A}^{-1}$ and $>600 \mathrm{~cd} \mathrm{~m}^{-2}$ at $6 \mathrm{~V}$. We note that this single-layer TADF LEC performance is competitive with that of multilayer OLEDs based on the same TADF polymer. We further show that the emission of the TADF polymer is highly sensitive to the proximity of the mobile ions, which are a fundamental constituent in LEC devices. This observation was utilized to shed light on the operation of TADF LEC devices, and we find that in hostguest TADF LECs, the excitons are distributed over the entire $\mathrm{p}-\mathrm{n}$ junction region, whereas in host-free TADF LECs the excitons appear to be strongly localized in close proximity to the doping regions. Our study thus identifies and clarifies an interesting interplay between the excitons and the mobile ions in TADF LECs and also provides a contribution to the development of sustainable emissive devices that can be entirely fabricated with low-energy and cost-efficient solution methods.

\section{EXPERIMENTAL METHODS}

The TADF polymer P1 $\left(M_{\mathrm{w}}=20500 \mathrm{~g} \mathrm{~mol}^{-1}\right)$ was synthesized by Yamamoto-type polymerization of the monomer (4-(3,6-dibromo-carbazol-9-yl)phenyl)(4-(dodecyloxy)phenyl)methanone using the procedure detailed in ref 42 . The host PYD-2Cz (409.48 $\mathrm{g} \mathrm{mol}^{-1}$, Lumtec), the electrolyte $\mathrm{THABF}_{4}\left(217.07 \mathrm{~g} \mathrm{~mol}^{-1}\right.$, Sigma-Aldrich), and the polymer PS $\left(M_{\mathrm{w}}=900000 \mathrm{~g} \mathrm{~mol}^{-1}\right.$ Sigma-Aldrich $)$ were used as received.

The master inks were prepared by separately dissolving the solutes in toluene at a concentration of 10 (P1) or $20 \mathrm{~g} \mathrm{~L}^{-1}$ (PYD-2Cz, $\mathrm{THABF}_{4}$ and PS) by stirring on a magnetic hot plate at $323 \mathrm{~K}$ for $>3 \mathrm{~h}$. The blend and active-material inks were prepared by blending the master inks in a desired mass ratio, followed by stirring on a magnetic hot plate at $323 \mathrm{~K}$ for $>3 \mathrm{~h}$. The ink-under-study was spin-coated (2000 rpm, 1000 $\mathrm{rpm} \mathrm{s} \mathrm{s}^{-1}, 60 \mathrm{~s}$ ) on a carefully cleaned substrate and thereafter dried at $343 \mathrm{~K}$ for $>2 \mathrm{~h}$. The thickness of the dry activematerial film was measured with a profilometer (DekTak XT, Bruker).

$\mathrm{CV}$ was carried out with a three-electrode setup equipped with a computer-controlled potentiostat (Autolab PGSTAT302, software: GPES). The working electrode comprised the material under study drop-cast on a Au-covered glass substrate, a $\mathrm{Pt}$ rod as the counter electrode, a $\mathrm{Ag}$ wire as the quasi-reference electrode, and $0.1 \mathrm{~mol} \mathrm{~L}^{-1}$ tetrabutylammonium hexafluorophosphate $\left(\mathrm{TBAPF}_{6}, \mathrm{Sigma}^{-A l d r i c h)}\right.$ in acetonitrile as the electrolyte solution. The scan rate was 0.05 $\mathrm{V} \mathrm{s}^{-1}$. Directly after each $\mathrm{CV}$ scan, a calibration scan was run with a small amount of ferrocene added to the electrolyte so that the $\mathrm{CV}$ potentials could be reported versus the ferrocene/ ferrocenium ion $\left(\mathrm{Fc} / \mathrm{Fc}^{+}\right)$reference potential. The reduction/ oxidation onset potentials were defined as the intersection of the baseline with the tangent of the current at the half-peak height. The CV preparation and characterization were executed within a $\mathrm{N}_{2}$-filled glovebox $\left(\left[\mathrm{O}_{2}\right]<1 \mathrm{ppm},\left[\mathrm{H}_{2} \mathrm{O}\right]\right.$ $<0.5$ ppm).

The optical transmission of $100 \mathrm{~nm}$ thick spin-coated thin films on quartz substrates (thickness $=1 \mathrm{~mm}$, Ted Pella) was measured with a spectrometer (C9920-02G, Hamamatsu Photonics), with a $100 \mathrm{~nm}$ thick PS film on a quartz substrate as a reference. The absorbance was estimated as [1 transmission], thus neglecting the effects of reflectance. The PL spectrum and the PLQY of the thin films were measured with an integrated sphere connected to the spectrometer under 
either an inert $\mathrm{N}_{2}$ atmosphere or an ambient-air atmosphere. The excitation wavelength in the PL and PLQY experiments was $300 \mathrm{~nm}$.

The ITO-coated glass substrates (ITO thickness $=160 \mathrm{~nm}$, $20 \Omega \mathrm{sq}^{-1}$, Thin Film Devices) were cleaned by a sequential 20 min ultrasonic treatment in detergent (Extran MA 01, Merck), distilled water, acetone (VWR), and isopropanol (VWR), drying at $393 \mathrm{~K}$ for $>4 \mathrm{~h}$, and UV-ozone exposure for $20 \mathrm{~min}$. PEDOT:PSS (, Clevios PVP AI 4083, Heraeus) was spincoated $\left(4000 \mathrm{rpm}, 1000 \mathrm{rpm} \mathrm{s}^{-1}, 60 \mathrm{~s}\right)$ on top of the ITO and thereafter dried at $393 \mathrm{~K}$ for $>2 \mathrm{~h}$. The thickness of the dry PEDOT:PSS film was measured to be $35 \mathrm{~nm}$.

The active-material ink was spin-coated either on top of the ITO or on top of the ITO/PEDOT:PSS and dried at $343 \mathrm{~K}$ for $>3 \mathrm{~h}$. The thickness of the active material was controlled by the spin-coating parameters $\left(800 \mathrm{rpm}, 800 \mathrm{rpm} \mathrm{s}^{-1}, 60 \mathrm{~s}\right)$. A 100 $\mathrm{nm}$ thick $\mathrm{Al}$ cathode was deposited on top of the active material by thermal vacuum evaporation at a base pressure below $2 \times 10^{-6}$ mbar. The emission area $2 \times 2 \mathrm{~mm}^{2}$ was defined by the overlap between the $\mathrm{Al}$ top cathode and the ITO bottom anode. All of the above procedures, with the exception of the PEDOT:PSS deposition, were carried out in two interconnected $\mathrm{N}_{2}$-filled glove boxes $\left(\left[\mathrm{O}_{2}\right]<1 \mathrm{ppm}\right.$, $\left.\left[\mathrm{H}_{2} \mathrm{O}\right]<0.5 \mathrm{ppm}\right)$.

The LEC devices were driven by and measured with a computer-controlled current-voltage-luminance system (OLED Lifetime Tester M6000, McScience), with the luminance measured in the forward direction. All LEC devices were characterized within $24 \mathrm{~h}$ of fabrication. The EL spectrum and intensity were measured with a carefully calibrated fiberoptic CCD-array spectrometer (Flame-S, Ocean Optics).

\section{ASSOCIATED CONTENT}

\section{SI Supporting Information}

The Supporting Information is available free of charge at https://pubs.acs.org/doi/10.1021/acs.jpclett.0c01506.

Energy levels of the neat host and guest films, optical properties of the neat host and guest films and the hostfree and host-guest active-material films, and additional LEC device performance data (PDF)

\section{AUTHOR INFORMATION}

\section{Corresponding Authors}

Qiang Wei - Ningbo Institute of Materials Technology \&

Engineering, Chinese Academy Sciences, Ningbo 315201, P. R. China; Email: weiqiang@nimte.ac.cn

Ludvig Edman - The Organic Photonics and Electronics Group, Department of Physics, Umeå University, SE-901 87 Umeå, Sweden; LunaLEC AB, SE-901 87 Umeå, Sweden; ๑ orcid.org/0000-0003-2495-7037; Email: ludvig.edman@ umu.se

\section{Authors}

Petter Lundberg - The Organic Photonics and Electronics Group, Department of Physics, Umeå University, SE-901 87 Umeå, Sweden

Ziyi Ge - Ningbo Institute of Materials Technology \& Engineering, Chinese Academy Sciences, Ningbo 315201, P. R. China; (1) orcid.org/0000-0003-3656-6017

Brigitte Voit - Leibniz-Institut für Polymerforschung Dresden e.V., DE-01069 Dresden, Germany; 이이이.org/0000-00024531-691X
Sebastian Reineke - Dresden Integrated Center for Applied Physics and Photonic Materials (IAPP), Institute for Applied Physics, Technische Universität Dresden, DE-01069 Dresden, Germany; 이이.org/0000-0002-4112-6991

Complete contact information is available at: https://pubs.acs.org/10.1021/acs.jpclett.0c01506

\section{Notes}

The authors declare no competing financial interest.

\section{ACKNOWLEDGMENTS}

We acknowledge the financial support from the Swedish Research Council, the Swedish Energy Agency, Carl Tryggers stiftelse, Kempestiftelserna, Stiftelsen Olle Engkvist Byggmästare, Bertil \& Britt Svenssons stiftelse för belysningsteknik, the National Natural Science Foundation of China (21805296, 21876158, 51773212, 21574144, and 21674123), Zhejiang Provincial Natural Science Foundation of China (LR16b040002), Natural Science Foundation of Ningbo City (2018A610134), and Zhejiang Provincial Natural Science Foundation of China (grant no. LY20B040002).

\section{REFERENCES}

(1) Yamanaka, T.; Nakanotani, H.; Hara, S.; Hirohata, T.; Adachi, C. Near-Infrared Organic Light-Emitting Diodes for Biosensing with High Operating Stability. Appl. Phys. Express 2017, 10, 074101.

(2) Chaves, M. E. D.; de Araujo, A. R.; Piancastelli, A. C. C.; Pinotti, M. Effects of Low-Power Light Therapy on Wound Healing: Laser X Led. An. Bras. Dermatol. 2014, 89, 616-623.

(3) Fischer, B.; Kreissl, S.; Boeffel, C.; Wedel, A. Multi-Layer Printing of Oleds as a Tool for the Creation of Security Features. Opt. Express 2012, 20, A219-A223.

(4) Choi, J.; Kanagaraj, S.; Choe, Y. Utilization of Novel Phenanthrene-Imidazole-Based Ionic Small Molecules for Blue Light-Emitting Electrochemical Cells. J. Mater. Chem. C 2020, 8, $4580-4587$.

(5) Sandström, A.; Edman, L. Towards High-Throughput Coating and Printing of Light-Emitting Electrochemical Cells: A Review and Cost Analysis of Current and Future Methods. Energy Technology 2015, 3, 329-339.

(6) Hu, S. Y.; Gao, J. Shaping Electroluminescence with a Large, Printed Bipolar Electrode Array: Solid Polymer Electrochemical Cells with over a Thousand Light-Emitting P-N Junctions. ChemElectroChem 2020, 7, 1748-1751.

(7) Yi, R. H.; Lo, C. L.; Luo, D.; Lin, C. H.; Weng, S. W.; Lu, C. W.; Liu, S. W.; Chang, C. H.; Su, H. C. Combinational Approach to Realize Highly Efficient Light-Emitting Electrochemical Cells. ACS Appl. Mater. Interfaces 2020, 12, 14254-14264.

(8) Youssef, K.; Li, Y.; O’Keeffe, S.; Li, L.; Pei, Q. B. Fundamentals of Materials Selection for Light-Emitting Electrochemical Cells. Adv. Funct. Mater. 2020, 1909102.

(9) Diethelm, M.; Grossmann, Q.; Schiller, A.; Knapp, E.; Jenatsch, S.; Kawecki, M.; Nüesch, F.; Hany, R. Optimized Electrolyte Loading and Active Film Thickness for Sandwich Polymer Light-Emitting Electrochemical Cells. Adv. Opt. Mater. 2019, 7, 1801278.

(10) Zhang, Z. T.; et al. A Colour-Tunable, Weavable Fibre-Shaped Polymer Light-Emitting Electrochemical Cell. Nat. Photonics 2015, 9, 233-238.

(11) Lanz, T.; Sandström, A.; Tang, S.; Chabrecek, P.; Sonderegger, U.; Edman, L. A Light-Emission Textile Device: Conformal SpraySintering of a Woven Fabric Electrode. Flexible and Printed Electronics 2016, 1, 025004.

(12) Liang, J. J.; Li, L.; Niu, X. F.; Yu, Z. B.; Pei, Q. B. Elastomeric Polymer Light-Emitting Devices and Displays. Nat. Photonics 2013, 7, 817-824. 
(13) Asadpoordarvish, A.; Sandström, A.; Larsen, C.; Bollström, R.; Toivakka, M.; Österbacka, R.; Edman, L. Light-Emitting Paper. Adv. Funct. Mater. 2015, 25, 3238-3245.

(14) Li, X. Y.; Gao, J.; Liu, G. J. Thickness Dependent Device Characteristics of Sandwich Polymer Light-Emitting Electrochemical Cell. Org. Electron. 2013, 14, 1441-1446.

(15) Hernandez-Sosa, G.; Tekoglu, S.; Stolz, S.; Eckstein, R.; Teusch, C.; Trapp, J.; Lemmer, U.; Hamburger, M.; Mechau, N. The Compromises of Printing Organic Electronics: A Case Study of Gravure-Printed Light-Emitting Electrochemical Cells. Adv. Mater. 2014, 26, 3235-3240.

(16) Sandström, A.; Dam, H. F.; Krebs, F. C.; Edman, L. Ambient Fabrication of Flexible and Large-Area Organic Light-Emitting Devices Using Slot-Die Coating. Nat. Commun. 2012, 3, 1002.

(17) Zimmermann, J.; Schlisske, S.; Held, M.; Tisserant, J.-N.; Porcarelli, L.; Sanchez-Sanchez, A.; Mecerreyes, D.; Hernandez-Sosa, G. Ultrathin Fully Printed Light-Emitting Electrochemical Cells with Arbitrary Designs on Biocompatible Substrates. Adv. Mater. Technol. 2019, 4, 1800641.

(18) Nuss, P.; Eckelman, M. J. Life Cycle Assessment of Metals: A Scientific Synthesis. PLoS One 2014, 9, No. e101298.

(19) Namanga, J. E.; Gerlitzki, N.; Mudring, A.-V. Scrutinizing Design Principles toward Efficient, Long-Term Stable Green LightEmitting Electrochemical Cells. Adv. Funct. Mater. 2017, 27, 1605588.

(20) Henwood, A. F.; et al. Blue-Emitting Cationic Iridium(Iii) Complexes Featuring Pyridylpyrimidine Ligands and Their Use in Sky-Blue Electroluminescent Devices. J. Mater. Chem. C 2017, 5, 9638-9650.

(21) Bolink, H. J.; Cappelli, L.; Coronado, E.; Gratzel, M.; Orti, E.; Costa, R. D.; Viruela, P. M.; Nazeeruddin, M. K. Stable Single-Layer Light-Emitting Electrochemical Cell Using 4,7-Diphenyl-1,10-Phenanthroline-Bis(2-Phenylpyridine) Iridium(Iii) Hexafluorophosphate. J. Am. Chem. Soc. 2006, 128, 14786-14787.

(22) Costa, R. D.; Orti, E.; Bolink, H. J.; Monti, F.; Accorsi, G.; Armaroli, N. Luminescent Ionic Transition-Metal Complexes for Light-Emitting Electrochemical Cells. Angew. Chem., Int. Ed. 2012, 51, 8178-8211.

(23) Slinker, J. D.; Rivnay, J.; Moskowitz, J. S.; Parker, J. B.; Bernhard, S.; Abruna, H. D.; Malliaras, G. G. Electroluminescent Devices from Ionic Transition Metal Complexes. J. Mater. Chem. 2007, 17, 2976-2988.

(24) Fresta, E.; Monclus, M. A.; Bertz, M.; Ezquerro, C.; MolinaAldareguia, J. M.; Berenguer, J. R.; Kunimoto, M.; Homma, T.; Costa, R. D. Key Ionic Electrolytes for Highly Self-Stable Light-Emitting Electrochemical Cells Based on $\operatorname{Ir}($ Iii) Complexes. Adv. Opt. Mater. 2020, 8, 2000295.

(25) Segal, M.; Baldo, M. A.; Holmes, R. J.; Forrest, S. R.; Soos, Z. G. Excitonic Singlet-Triplet Ratios in Molecular and Polymeric Organic Materials. Phys. Rev. B: Condens. Matter Mater. Phys. 2003, $68,075211$.

(26) Reufer, M.; Walter, M. J.; Lagoudakis, P. G.; Hummel, A. B.; Kolb, J. S.; Roskos, H. G.; Scherf, U.; Lupton, J. M. Spin-Conserving Carrier Recombination in Conjugated Polymers. Nat. Mater. 2005, 4, $340-346$.

(27) Köhler, A.; Bässler, H. Triplet States in Organic Semiconductors. Mater. Sci. Eng., R 2009, 66, 71-109.

(28) Lundberg, P.; Tsuchiya, Y.; Lindh, E. M.; Tang, S.; Adachi, C.; Edman, L. Thermally Activated Delayed Fluorescence with $7 \%$ External Quantum Efficiency from a Light-Emitting Electrochemical Cell. Nat. Commun. 2019, 10, 5307.

(29) Zeng, W.; Lai, H.-Y.; Lee, W.-K.; Jiao, M.; Shiu, Y.-J.; Zhong, C.; Gong, S.; Zhou, T.; Xie, G.; Sarma, M.; Wong, K.-T.; Wu, C.-C.; Yang, C. Achieving Nearly 30\% External Quantum Efficiency for Orange-Red Organic Light Emitting Diodes by Employing Thermally Activated Delayed Fluorescence Emitters Composed of 1,8-Naphthalimide-Acridine Hybrids. Adv. Mater. 2018, 30, 1870033.

(30) Wong, M. Y.; Zysman-Colman, E. Purely Organic Thermally Activated Delayed Fluorescence Materials for Organic Light-Emitting Diodes. Adv. Mater. 2017, 29, 1605444.
(31) Uoyama, H.; Goushi, K.; Shizu, K.; Nomura, H.; Adachi, C. Highly Efficient Organic Light-Emitting Diodes from Delayed Fluorescence. Nature 2012, 492, 234-238.

(32) Wong, M. Y.; La-Placa, M.-G.; Pertegas, A.; Bolink, H. J.; Zysman-Colman, E. Deep-Blue Thermally Activated Delayed Fluorescence (Tadf) Emitters for Light-Emitting Electrochemical Cells (Leecs). J. Mater. Chem. C 2017, 5, 1699-1705.

(33) Penfold, T. J.; Dias, F. B.; Monkman, A. P. The Theory of Thermally Activated Delayed Fluorescence for Organic Light Emitting Diodes. Chem. Commun. 2018, 54, 3926-3935.

(34) Liu, Y.; Li, C.; Ren, Z.; Yan, S.; Bryce, M. R. All-Organic Thermally Activated Delayed Fluorescence Materials for Organic Light-Emitting Diodes. Nature Reviews Materials 2018, 3, 18020.

(35) Jhulki, S.; Cooper, M. W.; Barlow, S.; Marder, S. R. Phosphorescent and Tadf Polymers and Dendrimers in SolutionProcessed Self-Host Organic Light-Emitting Diodes: Structure Analysis and Design Perspectives. Materials Chemistry Frontiers 2019, 3, 1699-1721.

(36) Nikolaenko, A. E.; Cass, M.; Bourcet, F.; Mohamad, D.; Roberts, M. Thermally Activated Delayed Fluorescence in Polymers: A New Route toward Highly Efficient Solution Processable Oleds. Adv. Mater. 2015, 27, 7236-7240.

(37) Luo, J.; Xie, G.; Gong, S.; Chen, T.; Yang, C. Creating a Thermally Activated Delayed Fluorescence Channel in a Single Polymer System to Enhance Exciton Utilization Efficiency for BluishGreen Electroluminescence. Chem. Commun. 2016, 52, 2292-2295.

(38) Nobuyasu, R. S.; Ren, Z.; Griffiths, G. C.; Batsanov, A. S.; Data, P.; Yan, S.; Monkman, A. P.; Bryce, M. R.; Dias, F. B. Rational Design of Tadf Polymers Using a Donor-Acceptor Monomer with Enhanced Tadf Efficiency Induced by the Energy Alignment of Charge Transfer and Local Triplet Excited States. Adv. Opt. Mater. 2016, 4, 653-653.

(39) Lee, S. Y.; Adachi, C.; Yasuda, T. High-Efficiency Blue Organic Light-Emitting Diodes Based on Thermally Activated Delayed Fluorescence from Phenoxaphosphine and Phenoxathiin Derivatives. Adv. Mater. 2016, 28, 4626-4631.

(40) Yang, Y.; Wang, S.; Zhu, Y.; Wang, Y.; Zhan, H.; Cheng, Y. Thermally Activated Delayed Fluorescence Conjugated Polymers with Backbone-Donor/Pendant-Acceptor Architecture for Nondoped Oleds with High External Quantum Efficiency and Low Roll-Off. Adv. Funct. Mater. 2018, 28, 1706916.

(41) Wei, Q.; Ge, Z.; Voit, B. Thermally Activated Delayed Fluorescent Polymers: Structures, Properties, and Applications in Oled Devices. Macromol. Rapid Commun. 2019, 40, 1800570.

(42) Wei, Q.; et al. Conjugation-Induced Thermally Activated Delayed Fluorescence (Tadf): From Conventional Non-Tadf Units to Tadf-Active Polymers. Adv. Funct. Mater. 2017, 27, 1605051.

(43) Tang, S.; Sandström, A.; Lundberg, P.; Lanz, T.; Larsen, C.; van Reenen, S.; Kemerink, M.; Edman, L. Design Rules for Light-Emitting Electrochemical Cells Delivering Bright Luminance at 27.5\% External Quantum Efficiency. Nat. Commun. 2017, 8, 1190.

(44) Tang, S.; Buchholz, H. A.; Edman, L. On the Selection of a Host Compound for Efficient Host-Guest Light-Emitting Electrochemical Cells. J. Mater. Chem. C 2015, 3, 8114-8120.

(45) Pommerehne, J.; Vestweber, H.; Guss, W.; Mahrt, R. F.; Bassler, H.; Porsch, M.; Daub, J. Efficient Two Layer Leds on a Polymer Blend Basis. Adv. Mater. 1995, 7, 551-554.

(46) Son, K. S.; Yahiro, M.; Imai, T.; Yoshizaki, H.; Adachi, C. Analyzing Bipolar Carrier Transport Characteristics of DiarylaminoSubstituted Heterocyclic Compounds in Organic Light-Emitting Diodes by Probing Electroluminescence Spectra. Chem. Mater. 2008, 20, 4439-4446.

(47) Scholes, G. D. Long-Range Resonance Energy Transfer in Molecular Systems. Annu. Rev. Phys. Chem. 2003, 54, 57-87.

(48) Dexter, D. L. A Theory of Sensitized Luminescence in Solids. J. Chem. Phys. 1953, 21, 836-850.

(49) Li, Y.; Wei, Q.; Cao, L.; Fries, F.; Cucchi, M.; Wu, Z.; Scholz, R.; Lenk, S.; Voit, B.; Ge, Z.; Reineke, S. Organic Light-Emitting Diodes Based on Conjugation-Induced Thermally Activated Delayed 
Fluorescence Polymers: Interplay between Intra- and Intermolecular Charge Transfer States. Front. Chem. 2019, 7, 1-12.

(50) Northey, T.; Stacey, J.; Penfold, T. J. The Role of Solid State Solvation on the Charge Transfer State of a Thermally Activated Delayed Fluorescence Emitter. J. Mater. Chem. C 2017, 5, 1100111009 .

(51) Zhang, D. D.; Cai, M. H.; Zhang, Y. G.; Zhang, D. Q.; Duan, L. Sterically Shielded Blue Thermally Activated Delayed Fluorescence Emitters with Improved Efficiency and Stability. Mater. Horiz. 2016, $3,145-151$.

(52) Hosokai, T.; Noda, H.; Nakanotani, H.; Nawata, T.; Nakayama, Y.; Matsuzaki, H.; Adachi, C. Solvent-Dependent Investigation of Carbazole Benzonitrile Derivatives: Does the ${ }^{3}$ le $-{ }^{1}$ ct Energy Gap Facilitate Thermally Activated Delayed Fluorescence? J. Photonics Energy 2018, 8, 1.

(53) van Reenen, S.; Akatsuka, T.; Tordera, D.; Kemerink, M.; Bolink, H. J. Universal Transients in Polymer and Ionic Transition Metal Complex Light-Emitting Electrochemical Cells. J. Am. Chem. Soc. 2013, 135, 886-891.

(54) Fang, J.; Matyba, P.; Edman, L. The Design and Realization of Flexible, Long-Lived Light-Emitting Electrochemical Cells. Adv. Funct. Mater. 2009, 19, 2671-2676. 